\title{
REMARKS ON THE RIGIDITY AND STABILITY OF MINIMAL SUBMANIFOLDS
}

\author{
MAKOTO SAKAKI
}

(Communicated by Jonathan M. Rosenberg)

Abstract. We improve the pinching theorem of Simons and the stability theorem of Barbosa and do Carmo with an elementary method.

Simons [7] proved a pinching theorem for closed minimal submanifolds in the unit sphere, which led to an intrinsic rigidity result. In this note, using an elementary method, we improve his theorem and obtain a result which does not depend on the dimension of the ambient space.

Lemma. Let $M$ be an $m$-dimensional minimal submanifold in a space of constant curvature $a$. Let $A$ and $\Delta$ denote the second fundamental form and the Laplacian of $M$, respectively. Then $-\langle A, \Delta A\rangle \leq\{2-2 /(m-1)(m+2)\}|A|^{4}-$ $m a|A|^{2}$.

Proof. We use the argument of Chern, do Carmo and Kobayashi [3]. We assume that the ambient space is $n$-dimensional. Set $q=2^{-1} m(m+1)-1=$ $2^{-1}(m-1)(m+2)$. When $n \leq m+q$, the Lemma is included in [3]. So we assume that $n>m+q$ in the following. We make a pointwise argument at a point $p$ on $M$. Let $\left\{e_{1}, \ldots, e_{n}\right\}$ be an orthonormal basis for the tangent space of the ambient space at $p$ such that $e_{1}, \ldots, e_{m}$ are tangent to $M$. We shall make use of the following convention on the ranges of indices: $1 \leq i$, $j \leq m, m+1 \leq \alpha, \beta \leq n, m+1 \leq \xi, \eta \leq m+q$. Let $h_{i j}^{\alpha}$ be the components of $A$ with respect to the basis. Set $T_{\alpha \beta}=\sum_{i, j} h_{i j}^{\alpha} h_{i j}^{\beta}$ and $T_{\alpha}=T_{\alpha \alpha}$.

It is an elementary observation that at each point the dimension of the image of the second fundamental form of an $m$-dimensional minimal submanifold is at most $2^{-1} m(m+1)-1=q$. Thus we may choose $e_{m+1}, \ldots, e_{n}$ so that $h_{i j}^{\alpha}=0$ for $\alpha>m+q$. Let $V$ be a subspace of the normal space of $M$ at $p$ spanned by $e_{m+1}, \ldots, e_{m+q}$. We define a symmetric linear transformation $T$ of $V$ by $T\left(\sum_{\eta} v^{\eta} e_{\eta}\right)=\sum_{\xi, \eta} T_{\xi \eta} v^{\eta} e_{\xi}$, which is well defined. As $T$ is symmetric, we may change $e_{m+1}, \ldots, e_{m+q}$ so that the $(q \times q)$-matrix $\left(T_{\xi \eta}\right)$ is diagonal. Then

Received by the editors July 11, 1988 and, in revised form, November 17, 1988 .

1980 Mathematics Subject Classification (1985 Revision). Primary 53C40; Secondary 53C20, $53 \mathrm{~A} 10$. 
apparently the $(n-m) \times(n-m)$-matrix $\left(T_{\alpha \beta}\right)$ is diagonal. So we can see that the equation (3.5) and the inequality (3.7) of [3] are valid with respect to this basis. Therefore, we obtain

$$
\begin{aligned}
-\langle A, \Delta A\rangle & \leq 2 \sum_{\xi \neq \eta} T_{\xi} T_{\eta}+\sum_{\xi} T_{\xi}^{2}-m a|A|^{2}=2\left(\sum_{\xi} T_{\xi}\right)^{2}-\sum_{\xi} T_{\xi}^{2}-m a|A|^{2} \\
& \leq 2\left(\sum_{\xi} T_{\xi}\right)^{2}-\frac{1}{q}\left(\sum_{\xi} T_{\xi}\right)^{2}-m a|A|^{2}=\left(2-\frac{1}{q}\right)|A|^{4}-m a|A|^{2}
\end{aligned}
$$

(cf. the proof of Lemma 5.3.1 of [7]). Thus the proof is complete.

Referring to [7] and [3] with the Lemma, we obtain the following:

Theorem 1. Let $M$ be an m-dimensional closed minimal submanifold in the unit sphere. Suppose that the scalar curvature $S$ of $M$ satisfies $m(m-1)\left(2 m^{2}+\right.$ $m-8) / 2\left(m^{2}+m-3\right) \leq S \leq m(m-1)$. Then either (i) $S=m(m-1)$ and $M$ is totally geodesic, or (ii) $S=\frac{2}{3}$ and $M$ is the Veronese surface in a totally geodesic 4-sphere.

By use of the same argument as above, we can improve the results of Yau [8] and Pan [5]. For example:

Theorem 2. Let $M$ be an m-dimensional complete submanifold with parallel mean curvature in the unit sphere. Suppose that the second fundamental form $A$ of $M$ satisfies $\{3+\sqrt{m}-2 /(m-1)(m+2)\}|A|^{2} \leq m-\varepsilon$ for a positive constant $\varepsilon$. Then $M$ lies in a totally geodesic $(m+1)$-sphere.

Using the inequality of the Lemma for $m=2$, we can improve the stability theorem of Barbosa and do Carmo [2].

Theorem 3. Let $M$ be a minimal surface in the simply-connected space form of constant curvature $a$, and let $D$ be a simply-connected compact domain with piecewise smooth boundary on $M$. Let $A$ denote the second fundamental form of $M$. If $\int_{D}\left(|a|+|A|^{2} / 2\right) d M<4 \pi / 3$, then $D$ is stable.

Remark. (i) When $a \geq 0$, Theorem 3 is proved in a little different way (cf. [1], Hoffman and Osserman [4]).

(ii) The proof of Lemma 2.11 of [2] is incorrect. In the proof of the lemma, we may choose the basis so that the components $h_{i j}^{\alpha}$ satisfy

$$
\left(h_{i j}^{3}\right)=\left(\begin{array}{cc}
\lambda & 0 \\
0-\lambda
\end{array}\right), \quad\left(h_{i j}^{4}\right)=\left(\begin{array}{cc}
0 & \mu \\
\mu & 0
\end{array}\right), \quad\left(h_{i j}^{5}\right)=\cdots=\left(h_{i j}^{n}\right)=0
$$

for some $\lambda$ and $\mu$ (cf. the proof of the Lemma above). The lemma is shown with the help of this fact.

(iii) In a succeeding paper [6] we will generalize Theorem 3 for a general ambient space.

\section{ACKNOWLEDGMENT}

The author wishes to thank the referee for useful suggestions. 


\section{REFERENCES}

1. J. L. Barbosa and M. do Carmo, Stability of minimal surfaces and eigenvalues of the Laplacian, Math. Z. 173 (1980), 13-28.

2. J. L. Barbosa and M. do Carmo, Stability of minimal surfaces in spaces of constant curvature, Bol. Soc. Brasil. Mat. 11 (1980), 1-10.

3. S. S. Chern, M. do Carmo and S. Kobayashi, Minimal submanifolds of a sphere with second fundamental form of constant length, Functional analysis and related fields, Springer, Berlin, 1970, pp. 59-75.

4. D. Hoffman and R. Osserman, The area of the generalized Gaussian image and the stability of minimal surfaces in $S^{n}$ and $R^{n}$, Math. Ann. 260 (1982), 437-452.

5. Y. L. Pan, Pinching theorems of Simons type for complete minimal submanifolds in the sphere, Proc. Amer. Math. Soc. 93 (1985), 710-712.

6. M. Sakaki, Estimates on the stability of minimal surfaces and harmonic maps, J. Math. Soc. Japan (to appear).

7. J. Simons, Minimal varieties in Riemannian manifolds, Ann. of Math. 88 (1968), 62-105.

8. S. T. Yau, Submanifolds with constant mean curvature II, Amer. J. Math. 97 (1975), 76-100.

Department of Mathematics, Tokyo Institute of Technology, Oh-Okayama, MEGURO-KU, TOKYO 152, JAPAN 